\title{
Functionalized-AgNPs for Long-Term Stability and Its Applicability in the Detection of Manganese Ions
}

\author{
Van-Tuan Hoang $\mathbb{D}^{1,2,3}$ Mai Mai, ${ }^{1}$ Le Thi Tam, ${ }^{1}$ Ngoc Phan Vu, ${ }^{2,3}$ Nguyen Tien Khi, ${ }^{2,3}$ \\ Phuong Dinh Tam $\mathbb{D}^{4},{ }^{4}$ Tran Quang Huy, ${ }^{2,5}$ Anh-Tuan Le $\mathbb{D}^{2,4}$ Ngo Xuan Dinh, ${ }^{6}$ \\ and Vinh-Hoang Tran ${ }^{7}$ \\ ${ }^{1}$ Advanced Institute for Science and Technology (AIST), Hanoi University of Science and Technology (HUST), \\ $1^{\text {st }}$ Dai Co Viet Road, Hanoi, Vietnam \\ ${ }^{2}$ Phenikaa University Nano Institute (PHENA), Phenikaa University, Hanoi 12116, Vietnam \\ ${ }^{3}$ Faculty of Biotechnology, Chemistry and Environmental Engineering, Phenikaa University, Hanoi 12116, Vietnam \\ ${ }^{4}$ Faculty of Materials Science and Engineering, Phenikaa University, Hanoi 12116, Vietnam \\ ${ }^{5}$ Faculty of Electric and Electronics, Phenikaa University, Hanoi 12116, Vietnam \\ ${ }^{6}$ University of Transport Technology, Thanh Xuan District, Hanoi 12116, Vietnam \\ ${ }^{7}$ School of Chemical Engineering, Hanoi University of Science and Technology (HUST), $1^{\text {st }}$ Dai Co Viet Road, Hanoi, Vietnam
}

Correspondence should be addressed to Van-Tuan Hoang; tuan.hoangvan@phenikaa-uni.edu.vn and Anh-Tuan Le; tuan.leanh@ phenikaa-uni.edu.vn

Received 6 August 2019; Revised 2 December 2019; Accepted 3 January 2020; Published 7 February 2020

Academic Editor: Gyorgy Szekely

Copyright (C) 2020 Van-Tuan Hoang et al. This is an open access article distributed under the Creative Commons Attribution License, which permits unrestricted use, distribution, and reproduction in any medium, provided the original work is properly cited.

\begin{abstract}
In this study, silver nanoparticles (AgNPs) were functionalized by various molecules, including sodium borohydride $\left(\mathrm{NaBH}_{4}\right)$, polyhexamethylene biguanide hydrochloride (PHMB), and Tween 80 to investigate the long-term stabilization of AgNPs in an aqueous dispersion. PHMB-functionalized silver nanoparticles (AgNPs/PHMB) exhibited better stability than others and could be stored at ambient temperature for at least 180 days. In addition to creating stabilization based on the electrostatic repulsion, the use of PHMB helped to increase the degree of stability of the colloidal AgNPs for a long time owing to strong interactions between $\mathrm{Ag}$ atoms on AgNPs with nitrogen (N) positions in PHMB molecules. The formed bond led to improving maintenance ability of the electrostatic repulsion layer among independent nanoparticles. The applicability of the as-prepared AgNPs/PHMB was also examined for $\mathrm{Mn}^{2+}$ detection via a colorimetric approach. The calibration curve was found to be linear over the range of 0-100 mM with a correlation coefficient of 0.97 . The amine groups of PHMB brought out a cooperative effect to form of iontemplated chelation with $\mathrm{Mn}^{2+}$, which caused the aggregation of AgNPs/PHMB. This suggested that the AgNPs/PHMB could be used as a potential probe in the detection of $\mathrm{Mn}^{2+}$ ions. More importantly, the long-term stability of AgNPs/PHMB paved a great promising path to provide many further solutions for the producer in practical applications.
\end{abstract}

\section{Introduction}

Silver nanoparticles (AgNPs) possess many unique chemical and physical properties; therefore, AgNPs have been widely studied and exploited in various applications, including medical, home appliances, electronics, textiles, and cosmetics [1]. The demand for AgNPs in the world is projected to increase, with production estimated at around 320 tons per year [1,2]. However, the properties of AgNPs strongly depend on their size, shape, and crystallinity. Moreover, large specific surface area and high surface energy lead to the self-aggregation of AgNPs during storing time, which considerably decreases their activity as well as applicability. These indicate that the stabilization of colloidal AgNPs is one of the most essential factors for developing practical applications [3-5]. Furthermore, the use of suitable stabilizers not only helps prevent the aggregation of AgNPs but also increases their activity. Therefore, many recent efforts have been performed to functionalize AgNPs by different stabilizers such as polymer or functional molecules, which contain amine 
groups/carboxylic acid groups/or thiol groups, etc., and due to these, the functional groups can form coordination bonding and/or electrostatic interactions with AgNPs [6-8].

Especially, herein, the polymer, which has many functional groups and high viscosity, has been considered as a promising stabilizer for the synthesis of AgNPs. Of the stabilizers used, the ionic stabilizers showed better stability than nonionic stabilizers. When silver nanoparticles are modified by an anionic stabilizer, their surface will electrically be charged. This leads to enhancing electrostatic repulsion among them, and as a result, the aggregation of colloidal AgNPs can be prevented [8]. Therefore, the cationic polymers such as polyhexamethylene biguanide hydrochloride (PHMB), chitosan (CS) [6,9], and polyethylenimine (PEI) [10], with molecule structures described in Figures 1(a)-1(c), respectively, are often used for stabilization of AgNPs. In such polymers, polyhexamethylene biguanide hydrochloride (PHMB), a surface-active agent, positively charged, highly biocompatible, and has been used as a disinfectant in medical, textile, and food industries [11,12], can stabilize the size and increase the dispersion of AgNPs. PHMB preserves the colloidal AgNPs for better stability and uniform dispersibility in the liquid media. Previous studies [11,13] showed that the PHMB-stabilized cationic silver nanoparticles displayed enhanced antimicrobial activity and demonstrated the promising potential for plant protection. However, the mechanism of durability and long-term stability of the colloidal AgNPs by ionic stabilizers may have been not fully understood.

Manganese $(\mathrm{Mn})$ element is an essential element of living organisms. The existence of the Mn element plays a critical role in the metabolism of the body and other biological processes. However, when using foods, especially drinking water with high $\mathrm{Mn}^{2+}$ ions content will cause harm to the human body, especially diseases related to the nervous system [14]. Some recent studies have shown that the colloidal AgNP-based colorimetric sensors for Mn ion detection have offered good sensitivity $[15,16]$. However, these colloidal AgNPs had short-term stability or have to use many stabilizers, which hinder their widespread applications. Therefore, the synthesis of the colloidal AgNPs with both long-term stability and flexibility is sustainable for practical application.

In this work, the AgNPs were functionalized by PHMB for long-term stability. Mechanism of the long-term stabilization of the AgNPs/PHMB was discussed. The aggregation of AgNPs/PHMB can be prevented by the electrostatic repulsion among them and the formation of the Ag- $\mathrm{N}$ coordinate covalent bonds. Besides, the AgNPs/PHMB with long-term stability have also been harnessed as a probe for the detection of $\mathrm{Mn}^{2+}$ ions by the colorimetric assays. The amine groups of PHMB brought out a cooperative effect to form ion-templated chelation with $\mathrm{Mn}^{2+}$, which caused the aggregation of AgNPs/PHMB.

\section{Materials and Experimental}

2.1. Materials. Silver nitrate $\left(\mathrm{AgNO}_{3}, 99.9 \%\right)$, sodium borohydride $\left(\mathrm{NaBH}_{4}, 99 \%\right)$, polyhexamethylene biguanide hydrochloride (powder, PHMB 98\%), Tween 80 (polysorbate $80,99 \%$ ), $\mathrm{Mn}\left(\mathrm{NO}_{3}\right)_{2} \cdot 4 \mathrm{H}_{2} \mathrm{O}, \mathrm{Co}\left(\mathrm{NO}_{3}\right)_{2} \cdot 6 \mathrm{H}_{2} \mathrm{O}$, and $\mathrm{Ni}\left(\mathrm{NO}_{3}\right)_{2} \cdot 6 \mathrm{H}_{2} \mathrm{O}$ were purchased from Shanghai Chemical Reagent. Deionized water was used. All chemicals were used without further purification.

2.2. Synthesis of the Functionalized AgNPs. The AgNPs/ PHMB, AgNPs/Tween 80, and AgNPs/ $\mathrm{NaBH}_{4}$ were prepared by the chemical reduction method using $\mathrm{NaBH}_{4}$ as a reducing agent along with $\mathrm{PHMB}$ or Tween 80 as stabilizers according to the following procedure: $60 \mathrm{~mL}$ of $\mathrm{AgNO}_{3}$ $(1 \mathrm{mM})$ solution was added dropwise into a $30 \mathrm{~mL}$ of aqueous solution containing each stabilizer $(0.02 \mathrm{mg} / \mathrm{mL})$ under continuous stirring. After $2 \mathrm{~h}, 6 \mathrm{~mL}$ of sodium borohydride $\left(\mathrm{NaBH}_{4}\right)$ solution $(10 \mathrm{mM}, 1: 1$ to the molar ratio of $\mathrm{AgNO}_{3}$ ) was added dropwise into the mixture. The reduction process was carried out for $3 \mathrm{~h}$ (optimized synthesis time) at room temperature (RT). Stirring was required during the synthesis procedure. The formation of AgNPs was proposed, according to [17] equation (1). Finally, the asprepared functionalized-AgNPs were stored at room temperature in the dark until further use:

$$
\mathrm{AgNO}_{3}+\mathrm{NaBH}_{4} \longrightarrow \mathrm{Ag}+\frac{1}{2} \mathrm{H}_{2}+\frac{1}{2} \mathrm{~B}_{2} \mathrm{H}_{6}+\mathrm{NaNO}_{3} .
$$

2.3. Characterization. The crystalline structure of AgNPs was examined using X-ray diffraction (XRD, Bruker D5005) using $\mathrm{CuK} \alpha$ radiation $(\lambda=0.154 \mathrm{~nm})$ at a step of $0.02^{\circ}(2 \theta)$ at room temperature. The UV-Vis absorbance spectra of the colloidal AgNPs were recorded by using a DR6000 spectrophotometer, and $10 \mathrm{~mm}$ path length quartz cuvettes were used for the measurement of the spectra. The surface chemistry of the colloidal AgNPs was examined by using Fourier Transform Infrared Spectrophotometer (IRAffinity1S, Shimadzu).

2.4. Long-Term Stability Measurements. The as-prepared colloidal AgNPs were stored in glass bottles under ambient temperature in the absence of light and were characterized by UV-Vis spectra in few hours and after 180 days of storage. In brief, $0.5 \mathrm{ml}$ of the as-prepared colloidal AgNPs solution at $\mathrm{pH} 6.8$ was diluted with $9.5 \mathrm{ml}$ of the distilled deionized water. The UV-Vis absorbance spectra were recorded by a DR6000 spectrophotometer. In addition, the surface charge and particle size distributions of the colloidal AgNPs were also measured at 2 hours and 180 days of storage using a particle size analyzer (Zetasizer Ver. 6.20, Malvern Instruments) to study the long-term stability of the colloidal AgNPs.

2.5. Colorimetric Detection of $\mathrm{Mn}^{2+}$ Ions. For the detection of $\mathrm{Mn}^{2+}$ ions, $1 \mathrm{ml}$ of the as-prepared colloidal AgNPs at pH 6.8 was diluted 9 times by distilled deionized water. Then, $1 \mathrm{ml}$ of $\mathrm{Mn}^{2+}$ solution with various concentrations (0-100 mM) was added into $9 \mathrm{ml}$ diluted colloidal AgNPs. The $\mathrm{pH}$ of the solution was adjusted to within the range of 3.5 to 9.0 using acetic acid (0.05\%) and $\mathrm{NH}_{4} \mathrm{OH}(30 \%)$. The mixture was 


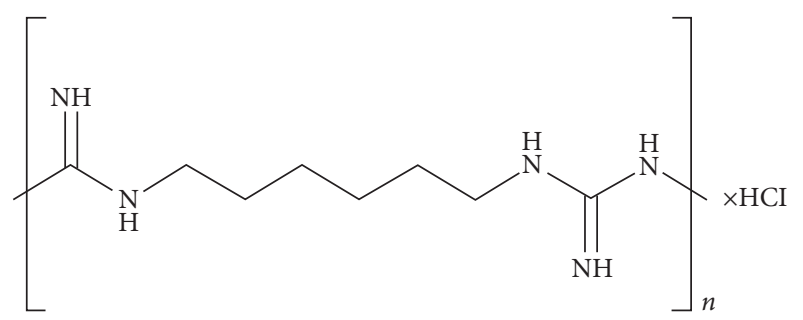

(a)

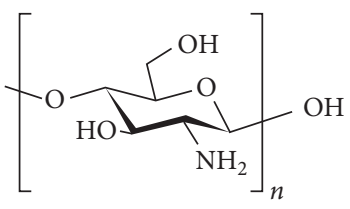

(b)

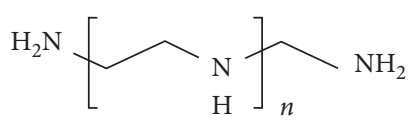

(c)

FIGURE 1: Chemical structure and functional groups of (a) polyhexamethylene biguanide hydrochloride (PHMB); (b) chitosan (CS), and (c) polyethylenimine (PEI).

kept in a shaker for 15 minutes at room temperature before the absorbance spectrum of this mixture was recorded. The limit of detection was calculated by using the following equation:

$$
\operatorname{LOD}=\frac{3 S}{b}
$$

where $S$ is the standard deviation of the blank solution and $b$ is the slope of the analytical curve.

\section{Results and Discussion}

3.1. Structural Analysis. Figure 2 shows the XRD spectra of colloidal AgNPs stabilized by PHMB (curve a), Tween 80 (curve b), and $\mathrm{NaBH}_{4}$ (curve c), respectively. It can be seen that four specific diffraction peaks of AgNPs at $2 \theta=38.1^{\circ}$, $44.3^{\circ}, 64.4^{\circ}$, and $77.5^{\circ}$ correspond to the crystals (111), (200), (220), and (311) of the silver metal crystal (JC PDS No. 040783). These results confirm the formation of the silver metal crystal with a relative single-phase structure in colloidal AgNPs. However, the intensity and sharpness of diffraction peaks in samples using various stabilizers are different. This indicates the stabilizer effect on the particle size of AgNPs as well as the quality of silver metal crystals. Line broadening in the pattern can be quantitatively evaluated using the Debye-Scherrer equation (equation (3)), which gives a relationship between peak broadening in XRD and particle size [18]:

$$
D=\frac{k \cdot \lambda}{\beta \cdot \cos \theta},
$$

where $D$ is the thickness of the crystal, $k$ is the DebyeScherrer constant (0.9), $\lambda$ is the X-ray wavelength $(0.15406 \mathrm{~nm}), \beta$ is the line broadening in radian obtained from the full width at half maximum, and $\theta$ is the Bragg angle. According to the Debye-Scherrer equation, average particle sizes of AgNPs were estimated to be 25, 20, and $15 \mathrm{~nm}$ when the used stabilizers were PHMB, Tween 80 , and $\mathrm{NaBH}_{4}$, respectively.

3.2. Long-Term Stability of the Colloidal AgNPs. UV-Vis can be used to observe the stabilization of AgNP colloids. Stable AgNP colloid in the UV-Vis spectra was stable vs. storing time, suggesting that particle size was stable and without any aggregation. The position of the plasmonic peak in the UV-

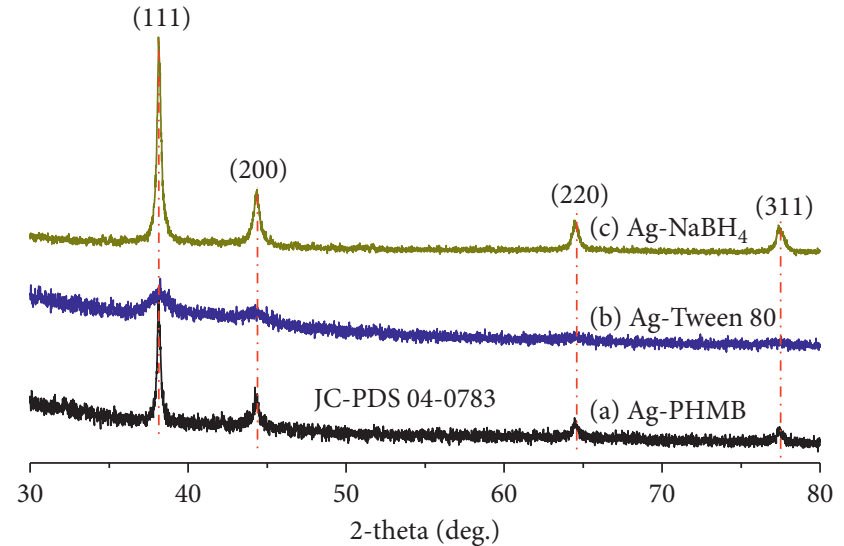

FIGURE 2: XRD diffraction pattern of the colloidal Ag-NPs stabilized by (a) PHMB, (b) Tween 80, and (c) $\mathrm{NaBH}_{4}$.

Vis spectrum depends on the average particle sizes and shapes [19]. Figure 3 shows the optical absorption spectra of the colloidal AgNPs with modified surfaces by different stabilizers as prepared (black solid line) and after 6 months of storage (red dash line). Figure 3 shows that as prepared, all synthesized AgNPs samples illustrated the appearance of the characteristic AgNP peaks at a maximum adsorption of $\lambda_{\max }=400 \mathrm{~nm}, 440 \mathrm{~nm}$, and $420 \mathrm{~nm}$, corresponding to $\mathrm{NaBH}_{4}, \mathrm{PHMB}$, and Tween 80 , respectively (black solid lines). However, after 180 days of storage, UV-Vis absorption spectra of these samples were decreased for all tested samples. The strongest decrease can be observed in the case of Tween 80 and $\mathrm{NaBH}_{4}$, respectively, and the peak absorption characteristic of silver nanoparticles was almost absent (red dash line in Figures 3(b) and 3(c)). In contrast, AgNP colloid was stabilized well by PHMB, and it still exists as the specific peak of AgNPs at $\lambda_{\max }=455 \mathrm{~nm}$ with a small reduction in absorption intensity. Clearly, this result indicated an excellent role of PHMB as a stabilizer to AgNP colloid than that of $\mathrm{NaBH}_{4}$ or Tween 80. Besides, in Figure 3(a), after 6 months, the specific peak of AgNPs is $\lambda_{\max }=455 \mathrm{~nm}$, and there is a small red shift, compared with $\lambda_{\max }=400 \mathrm{~nm}$ as-prepared samples, this can be attributed to a small aggregation that was formed in the AgNPs/PHMB system. The small aggregation in the AgNPs/PHMB system can also be observed by the DLS spectra (Figure 4). Two zones of size distribution as-prepared AgNPs/PHMB and small shoulder can be seen in Figure 4(a) with a mean particles size around $60 \mathrm{~nm}$. 


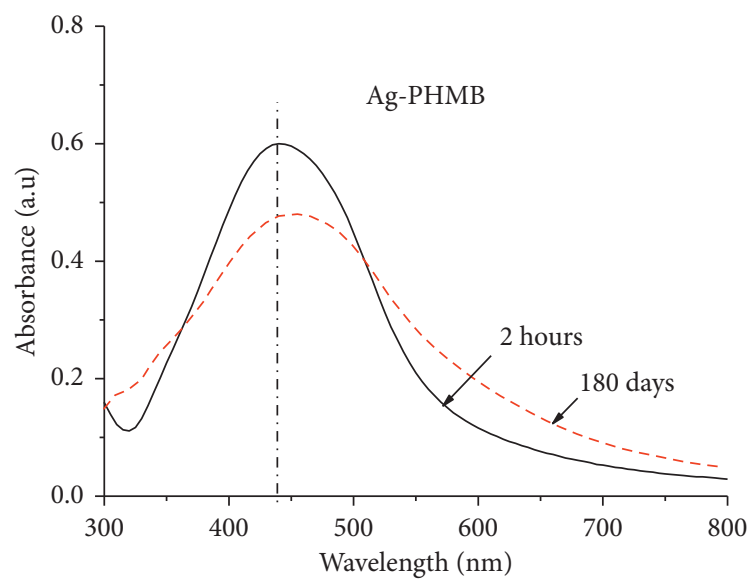

(a)

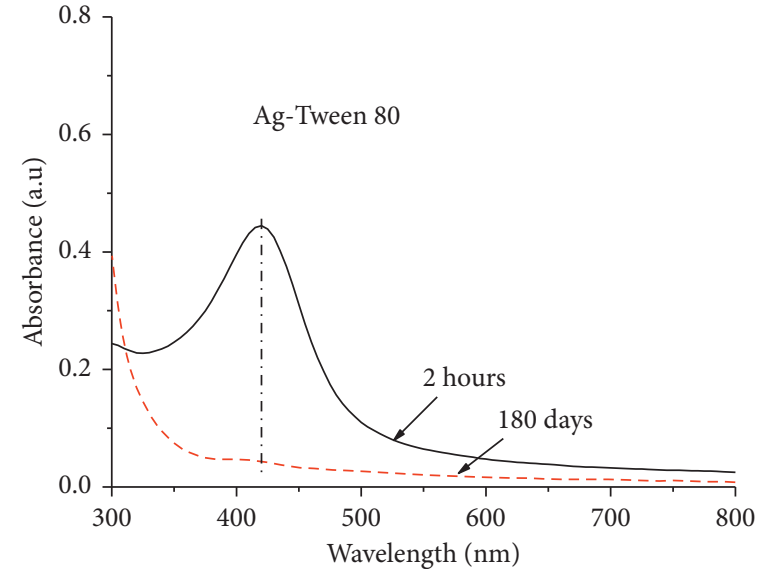

(b)

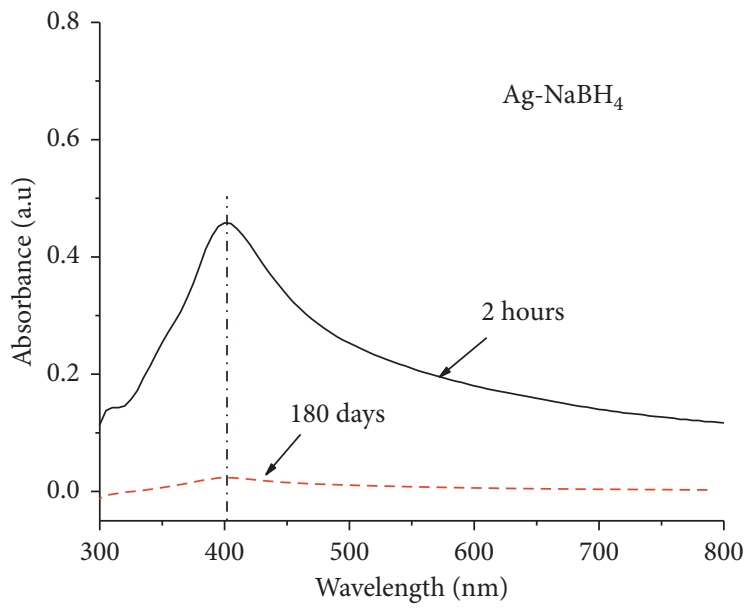

(c)

Figure 3: UV-Vis absorbance spectrum of the colloidal Ag-NPs stabilized by (a) PHMB, (b) Tween 80, and (c) $\mathrm{NaBH}_{4}$.

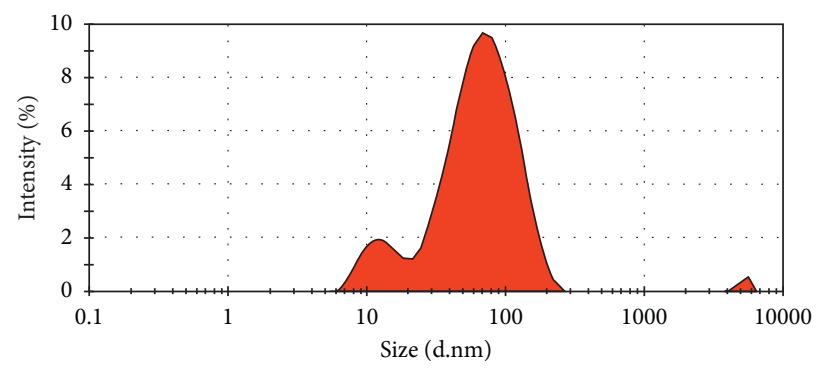

(a)

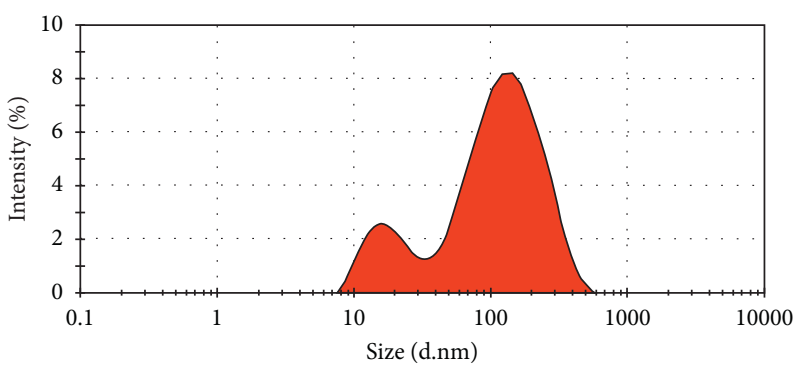

(b)

FIgUre 4: Size distribution for long term of the colloidal Ag-NPs stabilized by PHMB ((a) 2 hours; (b) 180 days).

After 6 months of storage, a shoulder was disappeared (Figure 4(b)); however, two zones of size distribution were still observed and the mean size was shifted to $105 \mathrm{~nm}$, which is bigger than that as prepared. These analyst results are completely consistent with the above UV-Vis measurements, as well as zeta potential measurements (Table 1). The surface charge of AgNP colloids with various used stabilizers was analysed by zeta potential measurements. The structure is formed by the surface interactions between AgNPs and stabilizer molecules which changes over time. In particular, Table 1 shows that the surface of modified AgNPs by ionic stabilizers (including $\mathrm{NaBH}_{4}$ and $\mathrm{PHMB}$ ) had less timevarying changes in zeta potential ( $\pm \%$ vs. storage time) than that of the nonionic stabilizer (i.e., Tween 80). After 180 days, AgNPs/PHMB showed better stability than the other stabilizers (Table 1). Moreover, the long-term stability of the colloidal AgNPs stabilized by PHMB when compared with other stabilizers or surfactants as shown in Table 2. In this 
TABLE 1: Effect of stabilizers on zeta potential after 180 days.

\begin{tabular}{lccc}
\hline \multirow{2}{*}{ Stabilizers } & & \multicolumn{2}{c}{ Zeta potential $(\mathrm{mV})$} \\
& 2 hours & 180 days & $\pm(\%)$ zeta potential change after 180 days \\
\hline PHMB & 31.5 & 26.5 & -15.87 \\
$\mathrm{NaBH}_{4}$ & -25.5 & -6.62 & -74.04 \\
Tween 80 & -7.9 & -0.02 & -99.75 \\
\hline
\end{tabular}

TABLe 2: Long-term stability of the colloidal AgNPs.

\begin{tabular}{lccccc}
\hline Methods & Precursors & Reducing agent & Stabilizer or surfactant & Stability time & Ref. \\
\hline Chemical synthesis & $\mathrm{AgNO}_{3}$ & $\mathrm{Citrate}$ & Citrate/gelatin mixture & 118 days & {$[3]$} \\
Chemical synthesis & $\mathrm{AgNO}_{3}$ & $\mathrm{NaBH}_{4}$ & Aminosilanes & days & {$[9]$} \\
Chemical synthesis & $\mathrm{AgNO}_{3}$ & $\mathrm{NaBH}_{4}$ & $\beta$-Cyclodextrin, adamantine & 14 days & {$[16]$} \\
Chemical synthesis & $\mathrm{AgNO}_{3}$ & $\mathrm{NaBH}_{4}$ & Dodecanethiol & 60 days & {$[7]$} \\
Green synthesis & $\mathrm{AgNO}_{3}$ & $\mathrm{NaBH}_{4}$ & L-Cysteine & 60 days & {$[20]$} \\
Biological synthesis & $\mathrm{AgNO}_{3}$ & Bacterial biomass & Bacterial biomass & 90 days & {$[21]$} \\
Chemical synthesis & $\mathrm{AgNO}_{3}$ & $\mathrm{NaBH}_{4}$ & PHMB & 180 days & This study \\
\hline
\end{tabular}

study, the colloidal AgNPs stabilized by PHMB for stability up to 180 days, which promises for many application fields, such as biomedical, agricultural, and environmental control.

3.3. Understanding the Mechanism of the Colloidal AgNPs Stabilized by PHMB. A mechanism for long-term stability of AgNPs stabilized by PHMB can be proposed as described in Figure 5. Based on the electron configuration of silver $\left(\mathrm{Ag}^{0}\right)$ is $[\mathrm{Kr}] 4 \mathrm{~d}^{10} 5 \mathrm{~s}^{1}$, there are many vacant orbitals on the covalent shell of silver atoms including $5 \mathrm{p}^{0} 5 \mathrm{~d}^{0}$. Meanwhile, there are many free-electron pairs (unbonded covalence electron pairs) at nitrogen $(\mathrm{N})$ positions on $\mathrm{PHMB}$ including - $\mathrm{NH}-7$ and/or $-\ddot{\mathrm{N}}$ = groups; therefore, coordination interaction of vacant-covalent orbitals of Ag with free-electron pairs of nitrogen in $\mathrm{PHMB}$ molecules has been generated, and as a result, the PHMB molecules protect AgNPs as modelled in Figure 5(a). More interestingly, the insidebonding structure of Ag into AgNPs with PHMB molecules are given in Figure 5(b), which suggested that AgNPs stabilized by $\mathrm{PHMB}$ were positively charged; therefore, in the AgNPs/PHMB, the stability of the electrostatic shield around AgNPs was enhanced by the formation of coordinate covalent bonds between the atoms of silver metal and nitrogen in the composition of PHMB (Figure 5(b)). According to this structure (Figure 5(b)), in the colloidal solution, AgNPs were separated from each other by the repulsion layer because of an electrostatic shield that protected the AgNPs surface in the form of positively charged spheres (Figure 5(c)). Thus, AgNPs tend to push each other and survive independently. Electrostatic repulsion of stabilizing agents on the surface of AgNPs is considered an important factor to improve the stability of the colloidal AgNPs [3].

The coordination interaction of vacant-covalent orbitals of Ag with free-electron pairs of nitrogen in PHMB molecules was further confirmed by the FTIR analysis. The FTIR spectra of PHMB and AgNPs/PHMB are shown in Figure 6. Compared with PHMB, the FTIR spectrum of AgNPs/ $\mathrm{PHMB}$ shows one new absorption peak at $1000 \mathrm{~cm}^{-1}$ which corresponds to $\mathrm{C}-\mathrm{N}$ bond stretching [22] and the disappearance of the absorption peak at $1556 \mathrm{~cm}^{-1}$ [11]. The occurrence of these changes indicates the interaction of the AgNPs with imine groups of PHMB through the formation of a coordination bond of Ag-N. The FTIR results demonstrate that the strong interactions of AgNPs and PHMB may efficiently prevent the aggregation of silver nanoparticles [23].

For $\mathrm{NaBH}_{4}$-stabilized colloidal AgNPs, the AgNPs were also stabilized by the charging shell covering the AgNPs. However, the electrostatic shield was created by the $\mathrm{BH}_{4}{ }^{-}$ anion and was broken as given in the following reaction:

$$
\mathrm{BH}_{4}{ }^{-}+4 \mathrm{H}_{2} \mathrm{O} \longrightarrow \mathrm{B}(\mathrm{OH})_{4}+4 \mathrm{H}_{2} \text {. }
$$

Therefore, the degradation of $\mathrm{BH}_{4}{ }^{-}$to hydrogen gas is the major cause of AgNPs to be aggregated [2,24]. With structure as shown in Figure 3(b), Tween 80 is a nonionic stabilizer; therefore, the interaction between Tween 80 and the AgNPs is based on the surface adsorption. The AgNPs surface was coated by a layer of Tween 80 to separate the nanoparticles and create great stability based on the steric repulsion mechanism $[8,25]$. Our finding results reveal that the long-term stability of the colloidal AgNPs was attributed to the formation of $\mathrm{Ag}-\mathrm{N}$ coordinate covalent bonds and the electrostatic repulsion between PHMB-functionalized AgNPs.

3.4. Application of AgNPs/PHMB as a Probe for the Detection of $\mathrm{Mn}^{2+}$ Ions. As described in Section 3.3, PHMB coated the AgNPs, therefore, AgNPs/PHMB particles contain freeamino groups, which were from a terminal tail of $\mathrm{PHMB}$ (Figure 7). The free $-\mathrm{NH}_{2}$ group can be used to recognize transition metal ions via a colorimetric approach, such as $\mathrm{Mn}^{2+}$ with the detection mechanism as shown in Figure 7. For $\mathrm{Mn}^{2+}$ detection, AgNP play the role to be a signal probe along with the free amino $\left(-\mathrm{NH}_{2}\right)$ group on PHMB molecules plays the role as a chemical capture probe. The presence of functional groups, such as amine $\left(-\mathrm{NH}_{2}\right)$, on the surface of AgNPs plays a critical role in the aggregation mechanism through metal-ligand interactions. The amino 


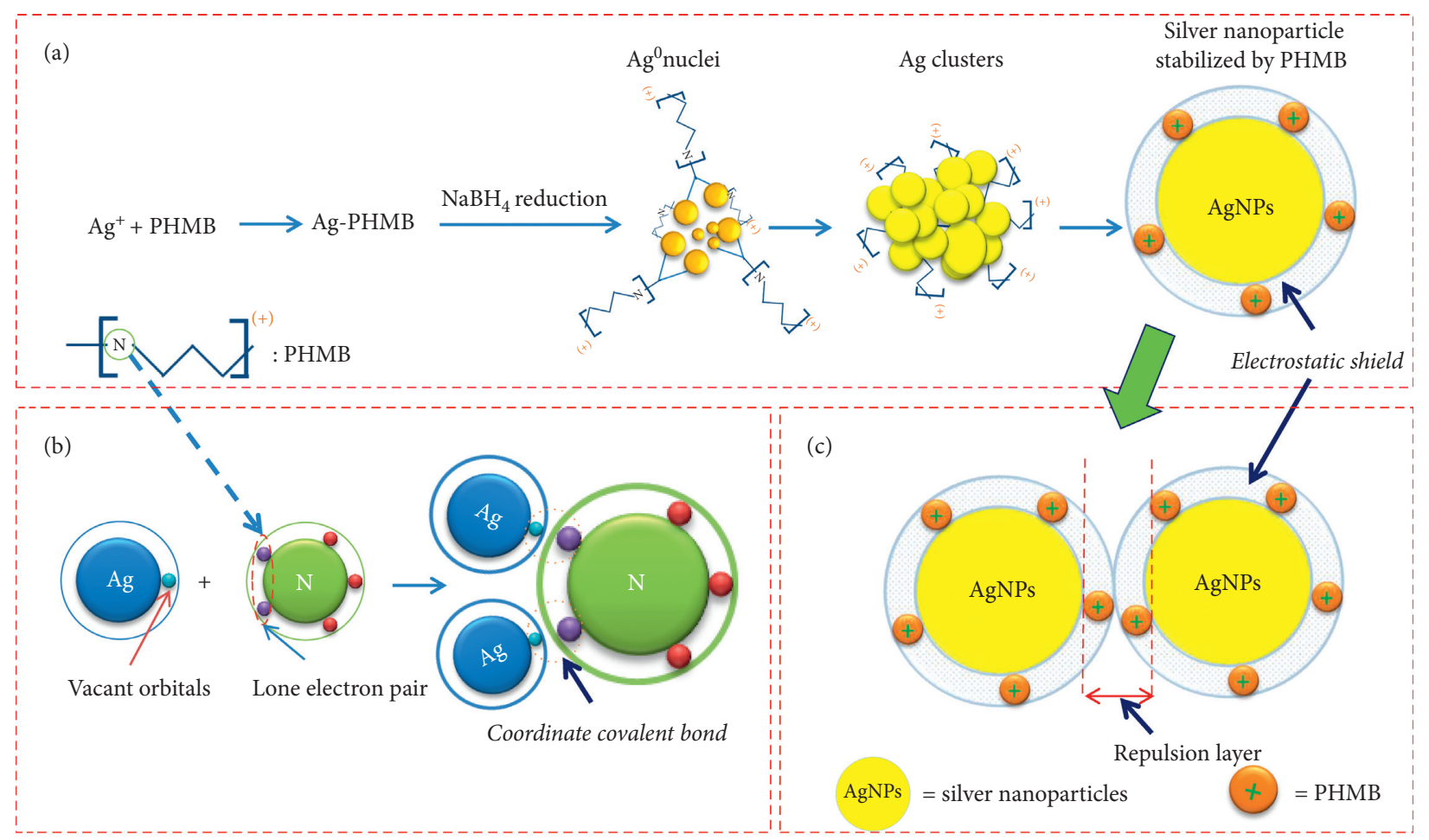

Figure 5: Schematic representation of AgNPs/PHMB: (a) $\mathrm{NaBH}_{4}$ reduction $\mathrm{Ag}^{+}$to AgNPs in the presence of PHMB; (b) the formation of the coordinate covalent bond between silver metal atoms $\left(\mathrm{Ag}^{0}\right)$ and free-covalence electron pairs of the nitrogen $(\mathrm{N})$ atom in $\mathrm{PHMB}$ molecules; and (c) mechanism stabilization of the AgNPs/PHMB via repulsion force AgNPs/PHMB particles.

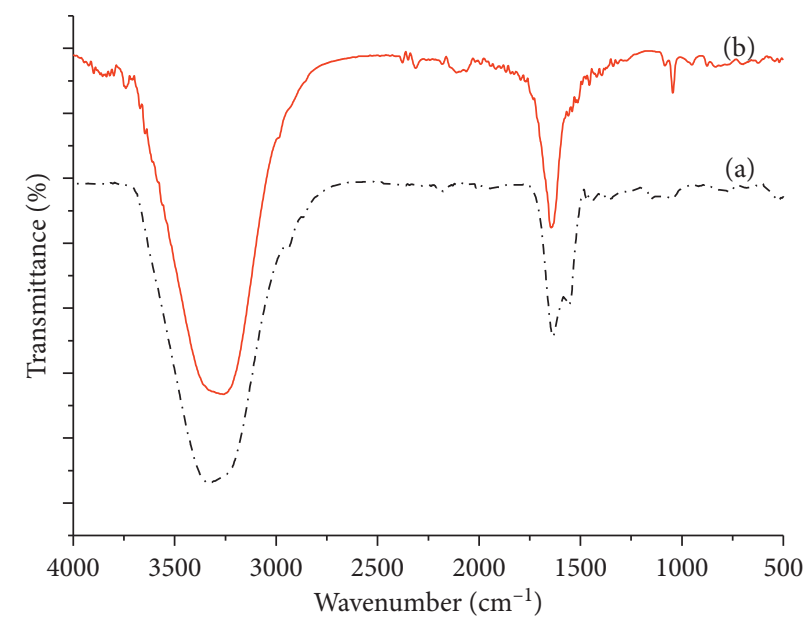

-... (a) PHMB

(b) Ag-PHMB

Figure 6: FTIR spectra (a) PHMB and (b) AgNPs stabilized by PHMB (AgNPs/PHMB).

groups of PHMB have a cooperative effect to form iontemplated chelation with $\mathrm{Mn}^{2+}$, which causes the aggregation of AgNPs [15]. On the contrary, the AgNP aggregation is induced by interparticle bond formation (cross-linking aggregation). The chelation form changes the interparticle distances of AgNPs, leading to the shift of the absorption peak of AgNPs to a longer wavelength. Therefore, cross- linking aggregation has been used for the detection of metal ions [26].

For that, control testes have been done based on adding $10 \mathrm{mM}$ of various ions including $\mathrm{Ni}^{2+}, \mathrm{Co}^{2+}$, and $\mathrm{Mn}^{2+}$ into the AgNPs/PHMB solution. The UV-Vis spectra of the AgNPs/PHMB solution before and after adding a solution containing the above ions are shown in Figure 8(a). It can 


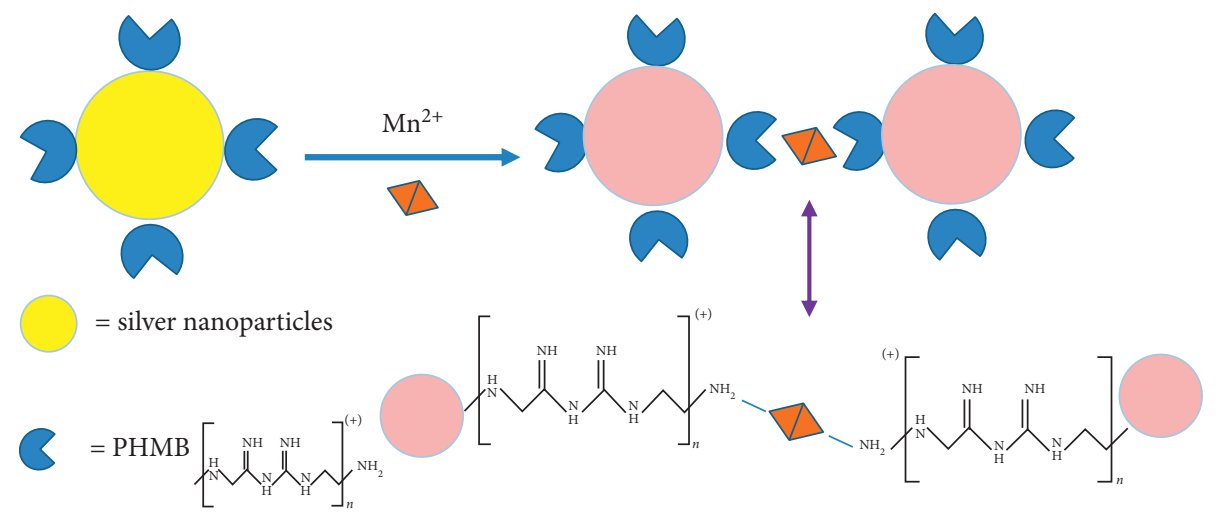

Figure 7: Mechanism of $\mathrm{Mn}^{2+}$-induced AgNPs/PHMB aggregation.

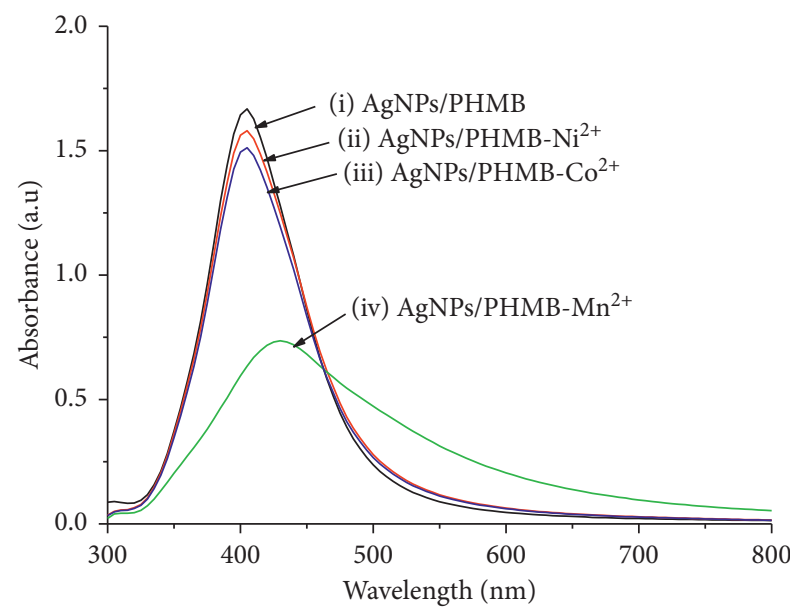

(a)

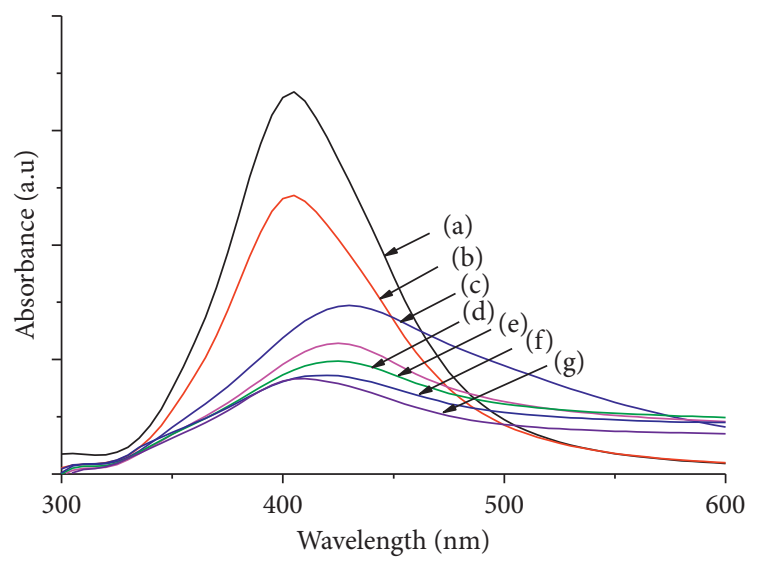

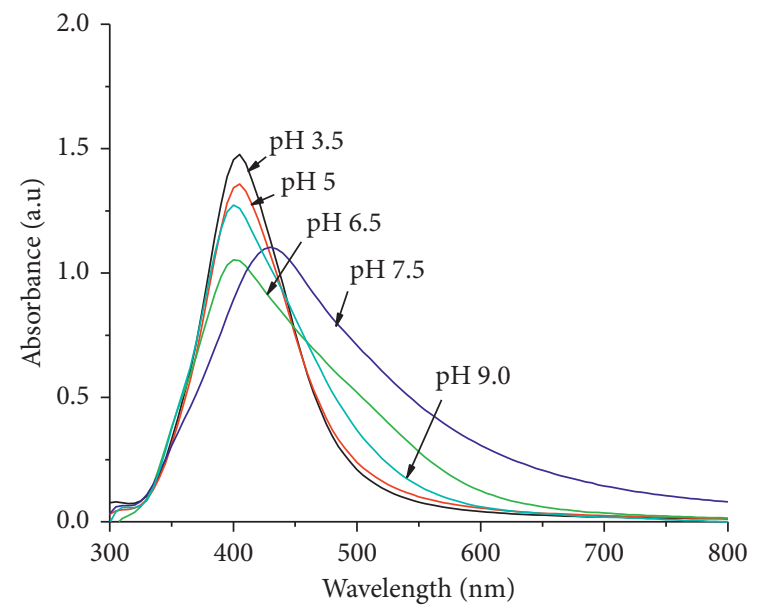

(b)

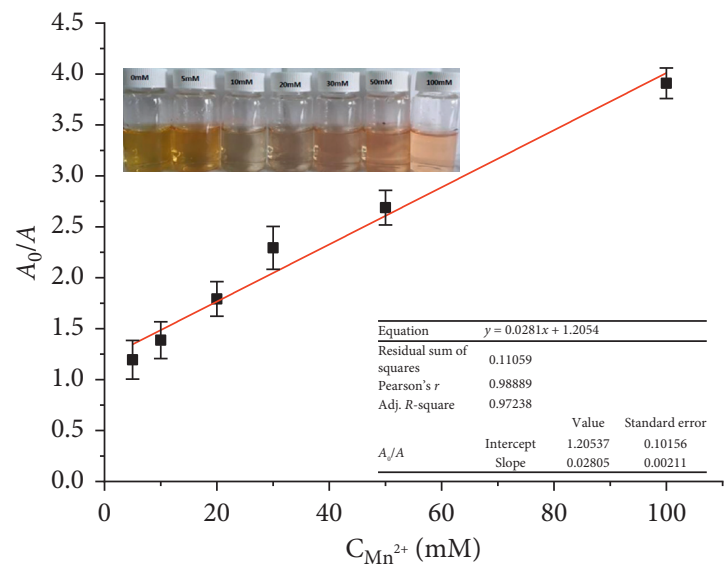
- (a) $0 \mathrm{mM}$
- (b) $5 \mathrm{mM}$
- (c) $10 \mathrm{mM}$
(d) $20 \mathrm{mM}$
(e) $30 \mathrm{mM}$
(f) $50 \mathrm{mM}$
(g) $100 \mathrm{mM}$

(c) (d)

Figure 8: Colorimetric detection of $\mathrm{Mn}^{2+}$ ions using the colloidal Ag-NPs: (a) UV-Vis spectra of AgNPs/PHMB without and with adding $\mathrm{Co}^{2+}, \mathrm{Ni}^{2+}$, and $\mathrm{Mn}^{2+}$ solution; (b) the UV-Vis spectra of AgNPs/PHMB solutions with different $\mathrm{pH}$ ranging from $\mathrm{pH} 3.5$ to $\mathrm{pH} 9.0$ after adding $10 \mathrm{mM} \mathrm{Mn}^{2+}$ solution; (c) UV-Vis spectra of AgNPs/PHMB in the presence of $\mathrm{Mn}^{2+}$ with different concentrations from 0 to $100 \mathrm{mM}$; and (d) a calibration curve for $\mathrm{Mn}^{2+}$ detection. 
be seen that the maximal absorption peak of the AgNPs/ PHMB solution after adding the $\mathrm{Mn}^{2+}$ ion (curve iv) was strongly diminished than the original AgNPs/PHMB solution (curve i), as well as this decrease was stronger than adding other ions such as $\mathrm{Ni}^{2+}$ (curve ii) or $\mathrm{Co}^{2+}$ (curve iii). These indicated the specific $\mathrm{Mn}^{2+}$ detection of the proposed method. The PHMB polymer can be used as a strong chelating agent to $\mathrm{Mn}$ (II) ions due to the amino groups which can form stable complexes with $\mathrm{Mn}(\mathrm{II})$ ions. The $\mathrm{N}$ atom and amino groups of the PHMB structure can form coordinated bonds with manganese ions causing the aggregation of large particles, which is in good agreement with similar previous reports $[15,16,26]$ in which the sulfonic group, hydroxyl group, and/or carboxyl groups have been considered as an important chelator in specific recognition with $\mathrm{Mn}^{2+}$ ions.

To investigate the effect of $\mathrm{pH}$ on the sensitivity of this colorimetric method to $\mathrm{Mn}^{2}+$, the AgNPs/PHMB solution was added with $10 \mathrm{mM} \mathrm{Mn}^{2+}$ solution at different $\mathrm{pH}$ (from $\mathrm{pH} 3.5$ to $\mathrm{pH}$ 9.0) and UV-Vis spectra are shown in Figure 8(b). It can be seen that when the $\mathrm{pH}$ value was less than 6.5, the UV-Vis spectra of the AgNPs/PHMB solution were unchanged after adding $\mathrm{Mn}^{2+}$ because the $-\mathrm{NH}_{2}$ group of PHMB is changed to $-\mathrm{NH}_{3}{ }^{+}$, a positively charged group; therefore, $\mathrm{Mn}^{2+}$ cannot interact to these positively charged groups. As the $\mathrm{pH}$ value was higher than $\mathrm{pH} \mathrm{8}$, it is noticed that the aggregation of AgNPs/ PHMB, as well as $\mathrm{Mn}^{2+}$, would be precipitated as the $\mathrm{Mn}(\mathrm{OH})_{2}$ form. At $\mathrm{pH} 6.5$ to $\mathrm{pH} 7.5$, the intensity and position of the maximum adsorption peaks changed more clearly; therefore, $\mathrm{pH}$ in the range 6.5 to 7.5 was selected for further experiments.

In the UV-Vis spectra of AgNPs/PHMB added with different $\mathrm{Mn}^{2+}$ concentrations (Figure $8(\mathrm{c})$ ), we notice that the intensity of the maximal absorption peak (at $\lambda_{\max }=403 \mathrm{~nm}$ ) was decreased with increasing $\mathrm{Mn}^{2+}$ ion concentrations. In addition, there was a red shift of this peak, which could be attributed to the cause of the AgNPs/PHMB aggregation via $\mathrm{Mn}^{2+}$ as linkers. The linear relationship between the absorbance intensity of the AgNPs/PHMB solution with $\mathrm{Mn}^{2+}$ concentration is shown in Figure 8(d) with the $\mathrm{Mn}^{2+}$ range from $0 \mathrm{mM}$ to $100 \mathrm{mM}$. The limit of detection (LOD) was calculated at approximately $0.22 \mathrm{mM}$. These finding results suggest that the AgNPs/PHMB also may be used as a useful probe for the detection of $\mathrm{Mn}^{2+}$ ions.

\section{Conclusions}

The long-term stability of colloidal AgNPs functionalized by $\mathrm{PHMB}, \mathrm{BH}_{4}^{-}$, and Tween 80 has been studied. The results indicated that the stability of AgNPs/PHMB (up to 180 days) was the best. The long-term stability of colloidal AgNPs is created by the formation of the coordinate covalent bonding as well as the enhancement of electrostatic repulsion between functionalized AgNPs. Moreover, the AgNPs/PHMB has been successfully tested for Mn(II) ion detection using the colorimetric assay. The AgNPs/PHMB aggregate in the presence of $\mathrm{Mn}^{2+}$, resulting in a color change of the solution as well as a red shift of the absorbance peak. The proposed method is capable of determining $\mathrm{Mn}^{2+}$ ions over a range of $0-100 \mathrm{mM}$. The assay is simple in design, fast in operation, low-cost, and can be observed with the naked eye. With long-term stability, the PHMB-functionalized AgNPs will become a promising material for many fields such as textile industry, agricultural, and environmental control.

\section{Data Availability}

The data used to support the findings of this study are available from the corresponding author upon request.

\section{Conflicts of Interest}

The authors declare that they have no conflicts of interest.

\section{Acknowledgments}

This research was funded by the Vietnam National Foundation for Science and Technology Development (NAFOSTED) under grant no. 103.99-2017.340.

\section{References}

[1] Q. H. Tran, V. Q. Nguyen, and A.-T. Le, "Silver nanoparticles: synthesis, properties, toxicology, applications and perspectives," Advances in Natural Sciences: Nanoscience and Nanotechnology, vol. 4, no. 3, p. 33001, 2013.

[2] B. Zewde, A. Ambaye, J. Stubbs III, and D. Raghavan, "A review of stabilized silver nanoparticles-synthesis, biological properties, characterization, and potential areas of applications," JSM Nanotechnology Nanomedicine, vol. 4, no. 2, p. 1043, 2016.

[3] J. Kavuličová, A. Mražíková, O. Velgosová, D. Ivánová, and M. Kubovčíková, "Stability of synthesized silver nanoparticles in citrate and mixed gelalin/citrate solution," Acta Polytechnica, vol. 58, no. 2, p. 104, 2018.

[4] A. M. E. Badawy, T. P. Luxton, R. G. Silva, K. G. Scheckel, M. T. Suidan, and T. M. Tolaymat, "Impact of environmental conditions ( $\mathrm{pH}$, ionic strength, and electrolyte type) on the surface charge and aggregation of silver nanoparticles suspensions," Environmental Science \& Technology, vol. 44, no. 4, pp. 1260-1266, 2010.

[5] A. Syafiuddin, Salmiati, M. R. Salim, A. Beng Hong Kueh, T. Hadibarata, and H. Nur, "A review of silver nanoparticles: research trends, global consumption, synthesis, properties, and future challenges," Journal of the Chinese Chemical Society, vol. 64, no. 7, pp. 732-756, 2017.

[6] H. V. Tran, L. D. Tran, C. T. Ba et al., "Synthesis, characterization, antibacterial and antiproliferative activities of monodisperse chitosan- based silver nanoparticles," Colloids and Surfaces A: Physicochemical and Engineering Aspects, vol. 360, no. 1-3, pp. 32-40, 2010.

[7] M. M. Oliveira, D. Ugarte, D. Zanchet, and A. J. G. Zarbin, "Influence of synthetic parameters on the size, structure, and stability of dodecanethiol-stabilized silver nanoparticles," Journal of Colloid and Interface Science, vol. 292, no. 2, pp. 429-435, 2005.

[8] L. Kvítek, A. Panáček, J. Soukupová et al., "Effect of surfactants and polymers on stability and antibacterial activity of silver nanoparticles (NPs)," The Journal of Physical Chemistry C, vol. 112, no. 15, pp. 5825-5834, 2008. 
[9] L. Cinteza, C. Scomoroscenco, S. Voicu et al., "Chitosanstabilized Ag nanoparticles with superior biocompatibility and their synergistic antibacterial effect in mixtures with essential oils," Nanomaterials, vol. 8, no. 10, p. 826, 2018.

[10] Z. Liu, Y. Wang, Y. Zu et al., "Synthesis of polyethylenimine (PEI) functionalized silver nanoparticles by a hydrothermal method and their antibacterial activity study," Materials Science and Engineering: C, vol. 42, pp. 31-37, 2014.

[11] S. Ashraf, N. Akhtar, M. Ghauri, M. Rajoka, Z. M. Khalid, and I. Hussain, "Polyhexamethylene biguanide functionalized cationic silver nanoparticles for enhanced antimicrobial activity," Nanoscale Research Letters, vol. 7, no. 1, p. 267, 2012.

[12] A. A. Gusev, A. A. Kudrinsky, O. V. Zakharova et al., "Versatile synthesis of PHMB-stabilized silver nanoparticles and their significant stimulating effect on fodder beet (Beta vulgaris L.)," Materials Science and Engineering: C, vol. 62, pp. 152-159, 2016.

[13] Y. A. Krutyakov and A. A. Kudrinsky, A. A. Gusev et al., Synthesis of positively charged hybrid PHMB-stabilized silver nanoparticles: the search for a new type of active substances used in plant protection products," Materials Research Express, vol. 4, no. 7, p. 75018, 2017.

[14] S. M. Rahman, M. Kippler, F. Tofail, S. Bölte, J. Derakhshani Hamadani, and M. Vahter, "Manganese in drinking water and cognitive abilities and behavior at 10 years of age: a prospective cohort study," Environmental Health Perspectives, vol. 125 , no. 5 , p. $57003,2017$.

[15] Y. Zhou, H. Zhao, C. Li et al., "Colorimetric detection of $\mathrm{Mn}^{2+}$ using silver nanoparticles cofunctionalized with 4-mercaptobenzoic acid and melamine as a probe," Talanta, vol. 97, pp. 331-335, 2012.

[16] R. Hu, L. Zhang, and H. Li, "A highly sensitive and selective colorimetric sensor for the detection of $\mathrm{Mn}^{2+}$ based on supramolecular silver nanoparticle clusters," New Journal of Chemistry, vol. 38, no. 6, pp. 2237-2240, 2014.

[17] A. C. Power, A. J. Betts, and J. F. Cassidy, "Silver nanoparticle polymer composite based humidity sensor," The Analyst, vol. 135 , no. 7, p. $1645,2010$.

[18] A. L. Patterson, "The scherrer formula for X-ray particle size determination," Physical Review, vol. 56, no. 10, pp. 978-982, 1939.

[19] I. V. Gmoshinski, S. A. Khotimchenko, V. O. Popov et al., "Nanomaterials and nanotechnologies: methods of analysis and control," Russian Chemical Reviews, vol. 82, no. 1, pp. 48-76, 2013.

[20] W. Zhang, L. Zhang, and Y. Sun, "Size-controlled green synthesis of silver nanoparticles assisted by L-cysteine," Frontiers of Chemical Science and Engineering, vol. 9, no. 4, pp. 494-500, 2015.

[21] A. T. M. Saeb, A. S. Alshammari, H. Al-Brahim, and K. A. AlRubeaan, "Production of silver nanoparticles with strong and stable antimicrobial activity against highly pathogenic and multidrug resistant bacteria," The Scientific World Journal, vol. 2014, Article ID 704708, 9 pages, 2014.

[22] R. J. Ouellette and J. D. Rawn, "Part I: functional groups and their properties," in Organic Chemistry, pp. 41-74, Elsevier, Amsterdam, Netherlands, 2014.

[23] A. L. Nogueira, R. A. F. Machado, A. Z. de Souza, F. Martinello, C. V. Franco, and G. B. Dutra, "Synthesis and characterization of silver nanoparticles produced with a bifunctional stabilizing agent," Industrial \& Engineering Chemistry Research, vol. 53, no. 9, pp. 3426-3434, 2014.

[24] L. Mulfinger, S. D. Solomon, M. Bahadory, A. V. Jeyarajasingam, S. A. Rutkowsky, and C. Boritz, "Synthesis and study of silver nanoparticles," Journal of Chemical Education, vol. 84, no. 2, p. 322, 2007.

[25] J. H. Lee, B. E. Park, Y. M. Lee, S. H. Hwang, and W. B. Ko, "Synthesis of fullereneC60-silver nanoparticles using various non-ionic surfactants under microwave irradiation," Current Applied Physics, vol. 9, no. 2, pp. e152-e156, 2009.

[26] M. Sabela, S. Balme, M. Bechelany, J.-M. Janot, and K. Bisetty, "A review of gold and silver nanoparticle-based colorimetric sensing assays," Advanced Engineering Materials, vol. 19, no. 12, Article ID 1700270, 2017. 